\title{
Variable optical/infrared counterpart to the transient gamma-ray source J0109+6134
}

\author{
E. Fernández-Valenzuela ${ }^{1}$, J. Martî2,4 ${ }^{2,}$ P. L. Luque-Escamilla ${ }^{3,4}$, A. J. Muñoz-Arjonilla ${ }^{4}$, and J. M. Paredes ${ }^{5}$ \\ ${ }^{1}$ Instituto de Astrofísica de Andalucía (CSIC), Glorieta de la Astronomía s/n, 18008 Granada, Spain \\ e-mail: estela@iaa.es \\ 2 Dept. de Física, EPSJ, Universidad de Jaén, Campus Las Lagunillas s/n, Edif. A3, 23071 Jaén, Spain \\ e-mail: jmarti@ujaen.es \\ 3 Dept. de Ingeniería Mecánica y Minera, EPSJ, Universidad de Jaén, Campus Las Lagunillas s/n, Edif. A3, 23071 Jaén, Spain \\ e-mail: peter@ujaen.es \\ ${ }^{4}$ Grupo de Investigación FQM-322, Universidad de Jaén, Campus Las Lagunillas s/n, Edif. A3, 23071 Jaén, Spain \\ e-mail: ajmunoz@ujaen.es \\ 5 Departament d'Astronomia i Meteorologia, Institut de Ciències del Cosmos, Universitat de Barcelona, IEEC-UB, \\ Martí i Franquès 1, 08028 Barcelona, Spain \\ e-mail: jmparedes@ub.edu
}

Received 26 July 2013 / Accepted 28 November 2013

\section{ABSTRACT}

\begin{abstract}
Context. We investigate the optical/infrared counterpart to the flaring gamma-ray source J0109+6134, which is believed to be a blazar seen through the Galactic plane.

Aims. The original aim of this work was to confirm the previously proposed optical counterpart by means of studying its temporal behaviour. The study was later extended to infrared wavelengths as new data became available.

Methods. We conducted a long-term differential CCD photometry campaign using the robotic Liverpool telescope. In addition, we used infrared satellite observations to also explore the source variability at these longer wavelengths.

Results. Evidence of variability well correlated with gamma-ray flares has been observed so far only in the infrared domain. This fact strongly supports that the proposed optical/infrared counterpart identification is correct. Moreover, our optical photometric campaign revealed an intense optical flare with 1.7 mag amplitude that occurs on time-scales of weeks. This optical event was observed to evolve without a counterpart in the nearly simultaneous gamma-ray monitoring by the Fermi satellite. Gamma-ray orphan optical flares have rarely been observed in other blazars, and J0109+6134 appears to be an interesting additional example for future studies.
\end{abstract}

Key words. gamma rays: galaxies - BL Lacertae objects: individual: J0109+6134

\section{Introduction}

The source J0109+6134, also known as GT0106+613, was originally discovered by Gregory \& Taylor (1981) during a radio patrol of the Galactic plane in search for variable radio sources using the National Radio Astronomy Observatory (NRAO) $91 \mathrm{~m}$ transit telescope. Follow-up observations with the NRAO Very Large Array (VLA) revealed an unresolved, highly polarised $(16 \%)$ source, showing both short-term and long-term radio variabilities with time-scales of days and years, respectively (Taylor $\&$ Gregory 1983). These facts were already indicative of a most likely extragalactic nature. Neutral hydrogen absorption spectra yielded a lower limit for the distance of $7.3 \mathrm{kpc}$ (Taylor \& Robert 1983).

The optical and infrarred counterpart was searched for by Paredes et al. (1993) and Norton et al. (1993), respectively. None of the proposed candidates was exactly consistent with the VLA radio position. The closest one was an optical stellar-like source with a Johnson magnitude $I=19.4$ and a 1.'2 offset.

In February 2010, the Fermi Large Area Telescope (LAT) detected J0109+6134 as a new flaring gamma-ray source (Vandenbroucke \& Hill 2010). This transient event was also partly covered in gamma- and X-rays by the AGILE and Swift observatories (Sabatini et al. 2010). Inspection of optical images from the IPHAS survey by Knigge et al. (2010) revealed a faint optical counterpart candidate (IPHAS J010946.33+613330.5) with magnitude $i^{\prime}=19.7$ and a much better match to the radio position than the one proposed in 1993. Optical spectroscopy by Vandenbroucke et al. (2010) with the Keck 1 telescope provided a blazar-like spectrum with an estimated redshift $z=0.783$. This fact strongly suggested a probable identification between the IPHAS and Fermi sources, since blazars are well-known gamma-ray emitters. However, the lack of correlated optical and gamma variability prevented confidence in the identification. At most, only some evidence for optical variability was present at time-scales of years in the IPHAS data alone, and when compared with the observations of Paredes et al. (1993).

In this paper, we present a long-term optical monitoring of the J0109+6134 IPHAS candidate that provides strong evidence for variability with large amplitude on time-scales of a week, and possibly shorter. These variations intriguingly occurred at an epoch well separated by hundreds of days with respect to the closest flaring gamma-ray episodes detected by Fermi LAT. Last but not least, we also report the source variability at infrared wavelengths. This part of our work is based on new satellite data that were not available at the time of the Fermi discovery and provide a high-confidence identification of the gamma-ray source counterpart. 


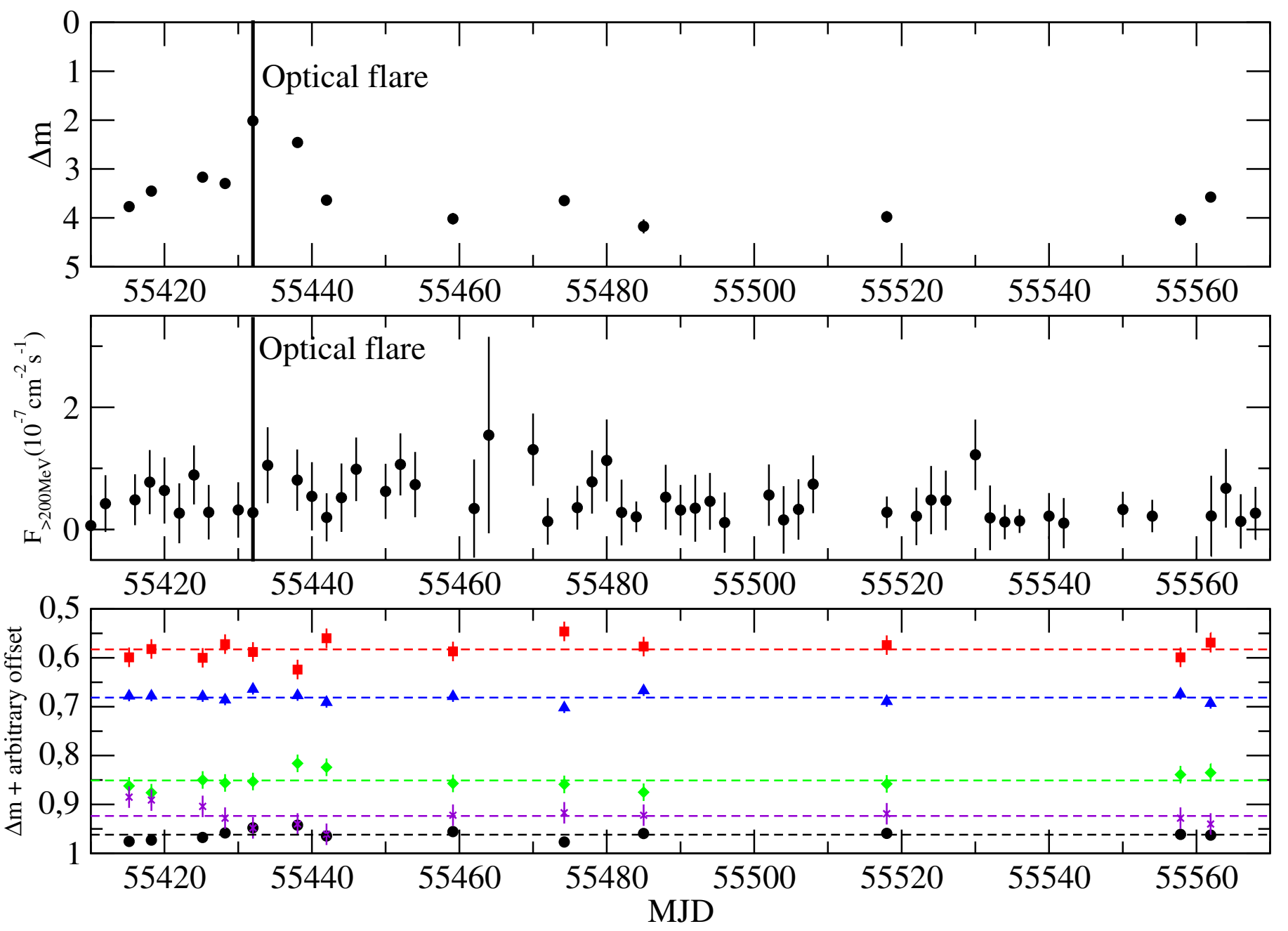

Fig. 1. Upper panel: differential light curve of the proposed blazar optical counterpart to J0109+6134/GT0106+613 in the $i$-band Sloan filter as a function of modified Julian day (MJD). The photometric points were obtained from the average of the different comparison stars. Error bars not shown are smaller than the symbol size. Middle panel: Fermi LAT light curve of J0109+6134 for energies above $200 \mathrm{MeV}$ based on an unbinned likelihood analysis with the Fermi science tools. Lower panel: photometric behaviour of comparison stars as a function of time. An arbitrary offset has been added to each of them for easier display. The dashed lines indicate the average of the differences of magnitude.

\section{Observations and results}

\subsection{Optical monitoring}

We carried out a photometric monitoring of the J0109+6134/ GT0106+613 field using the $2.0 \mathrm{~m}$ robotic Liverpool telescope (LT) at the Roque de los Muchachos observatory in La Palma (Canary Islands, Spain) ${ }^{1}$. A total of 39 photometric runs were obtained in the time interval between 6 August 2010 and 18 January 2011. The Ritchey-Chrétien focus of the telescope was used together with the RATCam instrument. This provides a field of view of $4^{\prime} .6 \times 4.6$ with a scale factor of $0 . \prime 135$ pixel $^{-1}$. The CCD frames were obtained through the $B$ and $V$ Bessell filters and the $r$ and $i$ Sloan filters. Typical exposure times were about $300 \mathrm{~s}$ under average seeing conditions of $0 .{ }^{\prime \prime} 9$.

After the first nights of observation, we decided to offset the pointing position of the telescope by a few arcminutes to avoid the bright $(V=7.7)$, high proper-motion star HD 6755. This action did not fully solve the problem. Even at the outside edge of the CCD field, the contamination introduced by HD 6755 still

\footnotetext{
1 http://telescope.livjm.ac.uk/
}

prevented us from accurately measuring the background level in photometric apertures.

To conduct differential photometry, we chose five isolated field stars with good photometric behaviour. Their magnitudes had to be brighter than the counterpart candidate J0109+6134 because this one often appeared at the detection limit of the telescope. Bias and flat-field correction were applied using the LT reduction pipeline, while the apphot package of the IRAF software ${ }^{2}$ was used to create the average differential-photometry light curve and its error, which depend on the flux, aperture- and background areas, standard deviation, and CCD parameters such as the adopted $e^{-}$to analogic data unit (ADU) factor. Circular apertures with radius adapted to seeing were always employed.

In the top panel of Fig. 1 and in Table 1, we present our final $i$-band light curve for the counterpart candidate proposed by Knigge et al. (2010). The data at other filters are not shown because the source was extremely faint and often undetectable at these shorter wavelengths. The stability of our differential photometry was judged by inspecting individual light curves of comparison stars as a function of time. They all remained flat and constant within $0.1 \mathrm{mag}$ or less. This number is acceptable considering the severe background problems mentioned above.

2 http://iraf.noao.edu/ 
Table 1. Optical monitoring of the proposed J0109+6134 counterpart in the $i$-band Sloan filter.

\begin{tabular}{cc}
\hline \hline Modified Julian day & Differential magnitude \\
\hline 55415.197 & $3.77 \pm 0.09$ \\
55418.221 & $3.45 \pm 0.06$ \\
55425.176 & $3.17 \pm 0.05$ \\
55428.218 & $3.30 \pm 0.06$ \\
55432.008 & $2.02 \pm 0.02$ \\
55438.048 & $2.46 \pm 0.04$ \\
55441.988 & $3.64 \pm 0.10$ \\
55459.133 & $4.02 \pm 0.12$ \\
55474.226 & $3.65 \pm 0.08$ \\
55485.004 & $4.18 \pm 0.14$ \\
55517.972 & $3.98 \pm 0.12$ \\
55557.834 & $4.04 \pm 0.12$ \\
55561.915 & $3.58 \pm 0.08$ \\
\hline
\end{tabular}

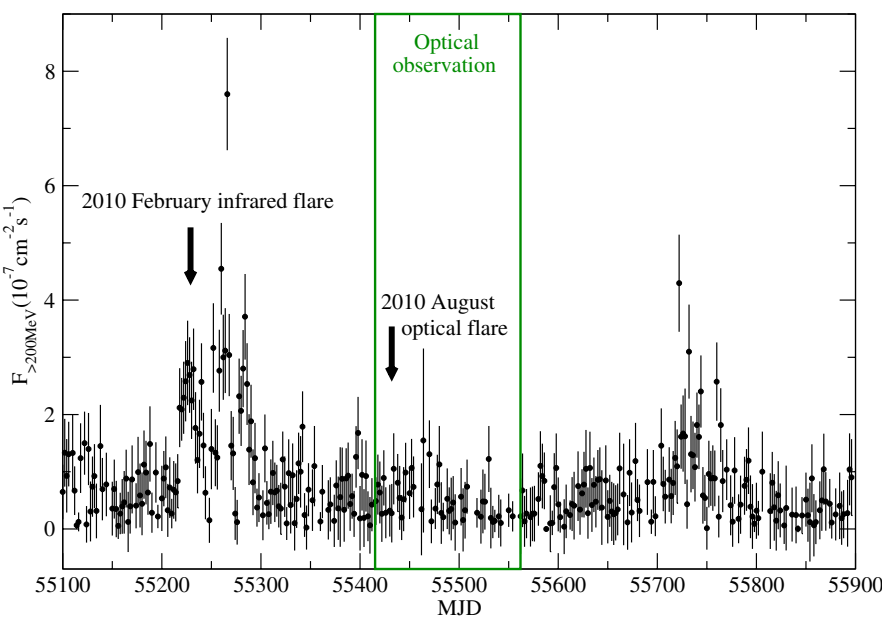

Fig. 2. Long-term Fermi LAT light curve of J0109+6134 for energies above $200 \mathrm{MeV}$ and covering a MJD time interval of about two years. No remarkable gamma-ray flares occurred during this time. The vertical arrows indicate the peaks of the optical and infrared flares reported here. The green lines represent the interval of our LT optical monitoring covered in Fig. 1.

For comparative purposes, the bottom pannel of Figs. 1 and 2 display the Fermi LAT ligthcurve for energies $E>200 \mathrm{MeV}$ using the Fermi Science Tools ${ }^{3}$. This light curve was obtained by means of unbinned likelihood analysis in a $10^{\circ}$ radius around the J0109+6134 position, with time bins of two days. The procedure is described in detail in Mattox et al. (1996), Abdo et al. (2010), and Nolan et al. (2012). The latest Fermi science tools version v9r $32 \mathrm{p} 5$ was used for this purpose. The background was accounted for by including the gal_2yearp7v6_v0 galactic diffuse model and the iso_p7v6source isotropic spectral template, as well as nearby sources in the Fermi LAT second source catalogue (Nolan et al. 2012). The instrument response function adopted was P7SOURCE_V6. A simple power-law energy spectrum was fitted to the target source to obtain its energy flux as a function of time.

\subsection{Far-infrared satellite data}

The Wide-field Infrared Survey Explorer (WISE) is a farinfrared satellite that performed an all-sky survey starting from its launch in 2009 (Wright et al. 2010). The instrument surveyed

\footnotetext{
3 http://fermi.gsfc.nasa.gov/ssc/
}
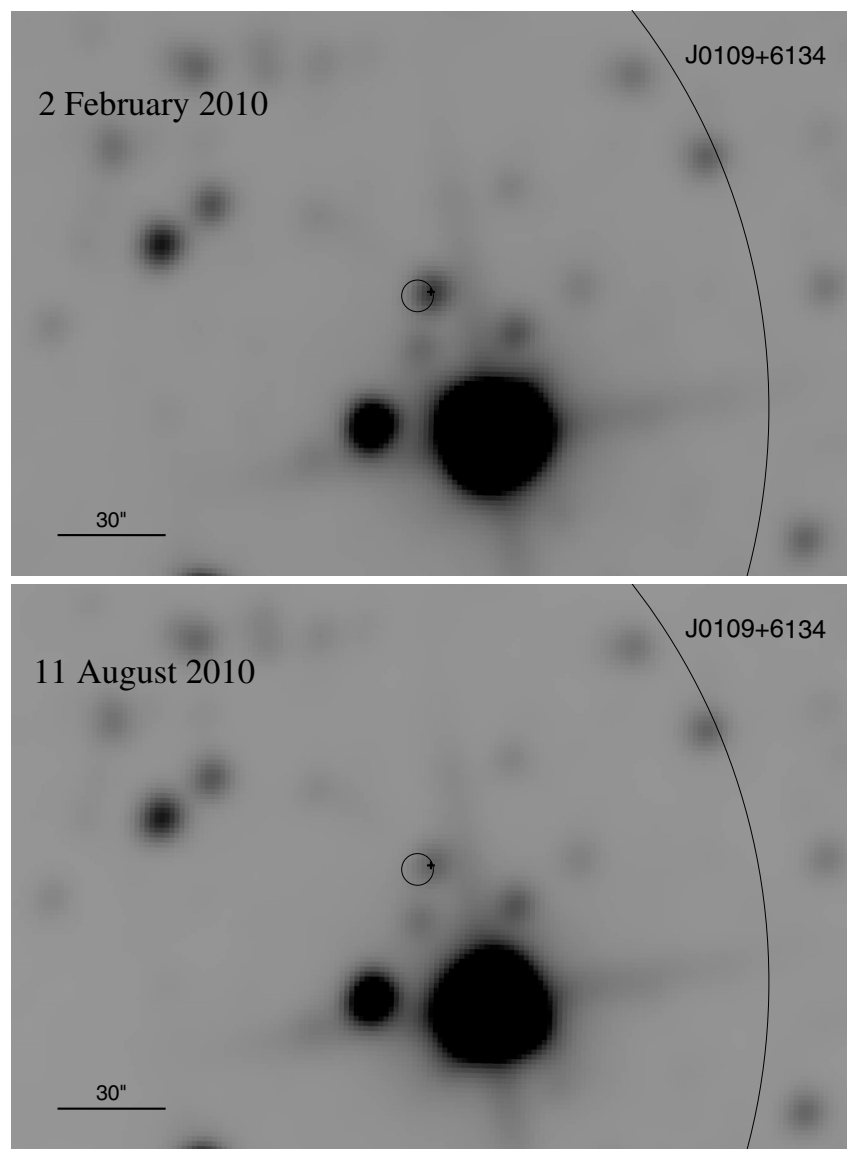

Fig. 3. Comparison of WISE frames covering the J0109+6134 field taken in 2010 February (top) and August (bottom), both in the $4.6 \mu \mathrm{m}$ filter. The brightness of the blazar counterpart has obviously changed in the time between the frames. The large arc is part of the J0109+6134 Fermi LAT error circle. The small circle and cross represent the Swift and Chandra X-ray positions, respectively. North is up and East is left.

the sky in four spectral bands (W1, W2, W3, and W4) corresponding to the $3.4,4.6,12$, and $22 \mu \mathrm{m}$ wavelengths. Its high-level data products can be directly downloaded from the WISE image service ${ }^{4}$.

WISE covered the J0109+6134 field on two occasions, with an observing time interval of about 5 days between the first and last frame acquisition. The resulting images are presented in Fig. 3 for the $4.6 \mu \mathrm{m}$ band. The first epoch was centred around 2 February 2010 (MJD 55 229.27), while the second one occurred six months later, centred around 11 August 2010 (MJD 55419.41). It is remarkable that the first date is almost in perfect coincidence with the strongest gamma-ray peak detected by Fermi LAT (see Fig. 2). In contrast, the second epoch corresponds to the early days of our LT optical monitoring when the gamma-ray source emitted at a quiescent level. The data quality was suitable for aperture photometry using IRAF to be used in the following section.

\section{Discussion}

From the observational point of view, blazars are well known to be highly variable active galactic nuclei in all bands of the electromagnetic spectrum. Flaring events in optical and gamma rays are often (but not always) well correlated with both positive

4 http://irsa.ipac.caltech.edu/applications/wise/ 
Table 2. IPHAS/WISE magnitudes of the proposed J0109+6134 counterpart available in the literature (Knigge et al. 2010; Wright et al. 2010).

\begin{tabular}{lcccccc}
\hline \hline Band & $r^{\prime}$ & $i^{\prime}$ & $W 1$ & $W 2$ & $W 3$ & $W 4$ \\
\hline Magnitude & 21.2 & 19.7 & $12.350 \pm 0.024$ & $11.238 \pm 0.021$ & $8.468 \pm 0.025$ & $5.998 \pm 0.039$ \\
\hline
\end{tabular}

and negative time-lags (see e.g., Ruan et al. 2012; Janiak et al. 2012; Sokolov et al. 2004; and Chatterjee et al. 2013). Typical delays observed are in the range from nearly zero to tens of days. The next obvious question here is whether any behaviour of this kind is observed in our case.

\subsection{Confirmation of the J0109+6134 optical/infrared counterpart}

The IPHAS source quoted in the introduction interestingly has an obvious correspondence with the infrared source WISE J010946.32+613330.4 in the WISE All-Sky Source Catalogue released in 2012. The WISE position uncertainty is about 0.'1 in each coordinate. This implies an almost perfect match with the similarly precise IPHAS coordinates, which strongly suggests that the two sources are actually the same object. The farinfrared magnitudes given in the WISE catalogue are compiled in Table 2 together with the rest of IPHAS data available in the literature.

Despite its scarce time coverage of two epochs only separated by six months, the WISE frames are crucial in providing suggestive evidence of correlated variability between the infrared and gamma-ray domain. Indeed, in the first epoch, which corresponds to the peak of the gamma-ray flare, the WISE source is 0.46 and 0.56 magnitudes brighter in the W2 and W3 bands, respectively, than in the second epoch with a low gamma-ray emission level (see Fig. 3). The W4 filter was not available in the second epoch, and W1 had background problems due to a bright diffraction spike of the nearby HD 6755. These significant brightness changes can be considered as a strong indication that the J0109+6134 optical/infrared counterpart identification is correct. Below, we proceed assuming that they are the same celestial source.

\subsection{Short-term optical variability in J0109+6134}

Moreover, our better-sampled optical photometric observations in Fig. 1 provide additional evidence of variability on time-scales of weeks and possibly shorter (few days). To estimate its statistical significance, we calculated the $C$ parameter defined as the ratio of the standard deviation of the object differential light curve and that of the control star as reported for instance by Jang \& Miller (1997), Howell et al. (1988), and Romero et al. (1999). According to these authors and references therein, if $C>4.155$, the observed variability can be considered real with a $99 \%$ confidence level. In our case, the value obtained amounts to $C=43.56$ and the variability is therefore beyond doubt. The two panels of Fig. 4 visually display this behaviour by comparing the two epochs with extreme brightness. The amplitude is as large as $\Delta m \simeq 1.7 \mathrm{mag}$. This short-term optical variability is reported here for the first time and reinforces the idea that IPHAS J010946.33+613330.5 is indeed the optical counterpart of the Fermi LAT source J0109+6134.

The observed optical flare in Fig. 1 peaked around MJD 55432 (23 August 2010), at a time when the Fermi LAT light curve remained relatively constant, with an average photon flux of $\sim 4 \times 10^{-7} \mathrm{ph} \mathrm{s}^{-1} \mathrm{~cm}^{-2}$. As can be seen in this

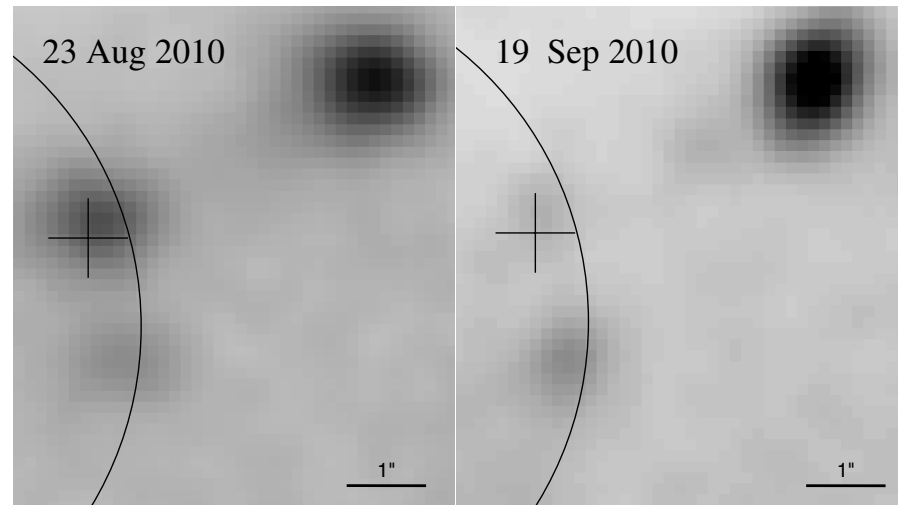

Fig. 4. Close-up comparison of the brightest and faintest states observed for IPHAS J010946.33+613330.5 during 2010 between 23 August (left) and 19 September (right). The two frames from the LT were taken using the Sloan $i$-band filter. The angular scale is indicated in the panel bottom-right corner. The proposed counterpart to J0109+6134 is the variable object consistent with the Chandra and Swift X-ray positions indicated by a cross and error circle, respectively. North is up and east is left.

figure and in the long-term Fermi LAT light curve shown in Fig. 2, there was no gamma-ray flare apparently associated with the optical one observed with the LT. The closest gamma-ray flaring events took place about 200 and 300 days before and after the 2010 August optical flare, respectively (see again Fig. 2). Therefore, the J0109+6134 optical counterpart flare appears to have no accompanying gamma-ray emission that could be associated with it. Although this behaviour may look anomalous, a similar lack of gamma-ray emission during optical/infrared flaring events has occasionally been observed before in other blazar sources. The case of PKS 0208-512 is a well-documented example (Chatterjee et al. 2013), and we may be facing a similar event in $\mathrm{J} 0109+6134$.

Chatterjee et al. (2013) proposed two different physical scenarios to interpret the absence of enhanced gamma-ray emision during optical flaring episodes of blazars. In a first one, the optical synchrotron emission is due to a change in the magnitude and/or direction of the magnetic field alone. The total number of relativistic electrons, their Doppler factor, and the number of seed photons available for inverse Compton scattering remain constant and so does the gamma-ray emission that depends on these parameters. The synchrotron flux is essentially proportional to $B^{2} \sin ^{2} \phi$, where $B$ is the magnetic field and $\phi$ is its angle with the line of sight. The $i$-band outburst amplitude of about 1.7 mag observed in our source corresponds to a brightness increase by a 4.7 factor. This translates into $B \sin \phi$ enhancement of 2.2. These values are very similar to those reported by Chatterjee et al. (2013) for PKS 0208-512.

In a second speculative scenario, the location of the outburst along the relativistic jet flow is the key factor. It has been shown that the ratio between inverse Compton and synchrotron emission is highly dependent on the location where the outburst takes place (Katarzyński \& Ghisellini 2007). For outbursts occurring very close to the central black hole, where the bulk Lorentz 
factor is still small, the optical to near infrared emission would significantly dominate the $\mathrm{GeV}$ band.

\section{Conclusions}

To conclude, we have reported the source WISE J010946.32+ 613330.4 as the infrared counterpart of the flaring gamma-ray source $\mathrm{J} 0109+6134$ based on its photometric variability, which is apparently correlated with the emission level observed by Fermi LAT. This fact appears well consistent with the blazar nature proposed by Vandenbroucke et al. (2010) and implies the confirmation of the previously proposed optical counterpart IPHAS J010946.33+613330.5 given the subarcsecond position coincidence between the WISE and IPHAS objects.

In addition, our optical monitoring of IPHAS J010946.33+ 613330.5 has revealed for the first time clear evidence of additional variability on time-scales of weeks. In particular, we observed a conspicuous $i$-band flare without a concurrent increase in the J0109+6134 gamma-ray flux. This type of gamma-ray orphan flares illustrates an unusual aspect of blazar physics, whose interpretation is still a matter of debate. The optical data reported here provide another example of this strange behaviour that complements that of the previously known PKS 0208-512.

Acknowledgements. The Liverpool telescope is operated on the island of La Palma by Liverpool John Moores University in the Spanish Observatorio del Roque de los Muchachos of the Instituto de Astrofísica de Canarias with financial support from the UK Science and Technology Facilities Council. This publication makes use of data products from the Wide-field Infrared Survey
Explorer, which is a joint project of the University of California, Los Angeles, and the Jet Propulsion Laboratory/California Institute of Technology, funded by the National Aeronautics and Space Administration. The authors acknowledge support by grants AYA2010-21782-C03-03 and AYA2010-21782-C03-01 from the Spanish Government, and Consejería de Economía, Innovación y Ciencia of Junta de Andalucía as research group FQM-322, as well as FEDER funds. J.M.P. also acknowledges financial support from ICREA Academia.

\section{References}

Abdo, A. A., Ackermann, M., Ajello, M., et al. 2010, ApJS, 188, 405 Chatterjee, R., Jorstad, S. G., Marscher, A. P., et al. 2008, ApJ, 689, 79 Chatterjee, R., Fossati, G., Urry, C. M., et al. 2013, ApJ, 763, L11

Ghisellini, G., Foschini, L., Tavecchio, F., et al. 2007, MNRAS, 382, L82 Gregory, P. C., \& Taylor, A. R. 1981, ApJ, 248, 596

Howell, S. B., Warnock, A., III, \& Mitchell, K. J. 1988, AJ, 95, 247

Janiak, M., Sikora, M., Nalewajko, K., et al. 2012, ApJ, 760, 129

Jang, M., \& Miller, H. R. 1997, AJ, 114, 565

Katarzyński, K., \& Ghisellini, G. 2007, A\&A, 463, 529

Knigge, C., Steeghs, D., Gaensicke, B., et al. 2010, ATel, 2429

Mattox, J. R., Bertsch, D. L., Chiang, J., et al. 1996, ApJ, 461, 396

Nolan, P. L., Abdo, A. A., Ackermann, M., et al. 2012, ApJS, 199, 31

Norton, A. J., Coe, M. J., Unger, S. J., et al. 1993, MNRAS, 260, 883

Paredes, J. M., Marti, J., Jordi, C., et al. 1993, A\&A, 102, 381

Romero, G. E., Cellone, S. A., \& Combi, J. A. 1999, A\&AS, 135, 477

Ruan, J. J., Anderson, S. F., MacLeod, C. L., et al. 2012, ApJ, 760, 51

Sabatini, S., Tavani, M., Bulgarelli, A., et al. 2010, ATel, 2416

Sokolov, A., Marscher, A. P., \& McHardy, I. M. 2004, ApJ, 613, 725

Taylor, A. R., \& Gregory, P. C. 1983, ApJ, 88, 1784

Taylor, G. B., \& Roberts, D. A. 1983, AJ, 106, 1087

Vandenbroucke, J., \& Hill, A. B. 2010, ATel, 2414

Vandenbroucke, J., Buehler, R., Ajello, M., et al. 2010, ApJ, 718, L166

Wrigth, E. L., Eisenhardt, P. R. M., Mainzer, A. K., et al. 2010, AJ, 140, 1868 